\title{
Hypothesis: The highly folded brain surface might be structured and located so as to facilitate inter- brain synchronization
}

\author{
Helen Minnis $\ddagger$, Maj-Britt Posserud§, Lucy Thompson‡, Christopher Gillbergl \\ ‡ University of Glasgow, Glasgow, United Kingdom \\ $\S$ Klinisk institutt 1, Bergen, Norway \\ | University of Gothenburg, Gothenburg, Sweden
}

Corresponding author: Helen Minnis (helen.minnis@glasgow.ac.uk)

Reviewable $\quad \mathrm{v} 1$

Received: 28 Nov 2019 | Published: 27 Feb 2020

Citation: Minnis H, Posserud M-B, Thompson L, Gillberg C (2020) Hypothesis: The highly folded brain surface might be structured and located so as to facilitate inter-brain synchronization. Research Ideas and Outcomes 6 : e48887. https://doi.org/10.3897/rio.6.e48887

\section{Abstract}

We integrate recent findings from neuro-anatomy, electroencephalography, quantum biology and social/neurodevelopment to propose that the brain surface might be specialised for communication with other brains.

Ground breaking, but still small-scale, research has demonstrated that human brains can act in synchrony and detect the brain activity of other human brains. Group aggregation, in all species, maximises community support and safety but does not depend on verbal or visual interaction. The morphology of the brain's outermost layers, across a wide range of species, exhibits a highly folded fractal structure that is likely to maximise exchange at the surface: in humans, a reduced brain surface area is associated with disorders of social communication. The brain sits in a vulnerable exposed location where it is prone to damage, rather than being housed in a central location such as within the ribcage.

These observations have led us to the hypothesis that the brain surface might be specialised for interacting with other brains at its surface, allowing synchronous non-verbal interaction. To our knowledge, this has not previously been proposed or investigated. 


\section{Keywords}

Brain surface, two-person neuroscience, brain-brain synchrony, quantum biological processes

\section{Overview and background}

In 2009, the gauntlet was thrown down for brain sciences to develop "two-person neuroscience" (Hari and Kujala 2009). Since then, a small and still controversial research base has begun to develop. Recent EEG studies have demonstrated brain-brain synchrony during human social interaction (Dumas et al. 2010), musical improvisation (Muller et al. 2013) and even between therapist and patient during music therapy (Fachner et al. 2019). EEG synchronicity has also been demonstrated in groups of high school students focussed on the same task (Dikker et al. 2017, Bevilacqua et al. 2019). A few studies have begun to use EEG to investigate interaction between pairs of individuals at a distance, under laboratory conditions that prevent sensory communication (Giroldini et al. 2015). The event-related potentials (ERPs) noted in "receivers" were small - much smaller than the ERPs of the "senders" - but statistically significant and synchronised with those of the "senders" despite the timing of sending being random (Giroldini and Pederzoli 2018). These intriguing findings - and our own observations about the brain's location and the morphology of its surface - lead us to propose the highly novel hypothesis below.

\section{Hypothesis}

The brain surface is ideally structured, and the brain is ideally located, to facilitate synchronous interaction between community members independent of sense organs.

\section{Objectives}

To present arguments for developing this novel perspective, with reference to recent data and ideas from various scientific fields.

To generate testable sub- hypotheses that we hope will stimulate integrative crossdisciplinary research about the role of the brain surface.

\section{Supporting Arguments}

\section{Highly folded surfaces in nature have a purpose}

Objects in nature that are specialised for a surface exchange function tend to have multiple units terminating at the surface, organised in a self-similar or fractal pattern. Such fractals are widely present in nature, from trees to mitochondria (West et al. 1999) and the purpose of their large surface area is usually to facilitate an exchange function at the surface with 
maximal metabolic capacity: photosynthesis in the leaves of trees or cell energy maintenance in mitochondria (Okie 2013). The brain surface is one of these highly folded structures (Batchelor et al. 2002) with a fractal geometry (Mota and Herculano-Houzel 2015). Both the cerebral and cerebellar surfaces have highly folded fractal folding patterns, but the purpose of this large, folded brain surface is still not fully understood. The traditional view is that folding within a fixed skull volume has the purpose of increasing cognitive capacity by increasing the amount of grey matter (Roth and Dicke 2005). This is undoubtedly true: surface folding maximises the number of cortical neurones and, hence, processing capacity, but this is unlikely to be the only reason for surface folding as there is no direct relationship between the degree of cortical folding and the number of neurones in the grey matter (Mota and Herculano-Houzel 2015). Objects in nature with an exchange function are not only highly folded but also have multiple repeating units at the surface (West et al. 1999). In the cerebellum, an example of a repeating unit terminating at the surface is the Purkinje cell. Cerebellar Purkinje cells have long axons, which, close to the cerebellar surface, branch into a rich network of dendrites that extend to cover a large surface area (Chaumont et al. 2013). Purkinje cells are involved in transmitting information from the cerebellar surface to deeper areas within the brain. Although their anatomy and physiology has been intensively studied, their precise functional role is still poorly understood (Tsubota et al. 2013). The cerebral cortex also has highly developed and even more complex connections across the cortical surface that are still being described, including several different types of interneurons with elaborate cross-connections (Jiang et al. 2015).

\section{Aggregation into herds, shoals and flocks does not depend on the senses or on leaders}

In advanced species, social groups support cooperative activities and protect against harm (Bowlby 1982). Although humans are the most advanced species in terms of social interaction, as evidenced by our sophisticated use of language and social networking, many other mammals also use social interaction to form and maintain herds. Movement in herd animals - including in human crowds - appears to be initiated by only a few individuals, and whether or not movement can be initiated by a particular individual is correlated with the degree of social interaction that individual has had with others in the group (Krueger et al. 2014). Yet group aggregation led by individuals does not explain everything: all species clump together in the face of danger and sometimes do so in a highly complex and unexplained manner (Liu et al. 2016). Across species, attachment behaviours are elicited at times of stress and result in members of social groups seeking close physical proximity with one another in order to achieve strength and protection in numbers (Bowlby 1982). The formation of herds, flocks or shoals, through aggregation of individuals of the same species, is a universal anti-predator mechanism that occurs across all species and taxa - from mammals to microbes (Liu et al. 2016). The process of aggregation therefore does not depend on language or sight, nor on the availability of a leader - although sensory information (Kimbell and Morrell 2015) and leadership (Andrieu et al. 2016) can enhance these processes. This poses a question as to whether there are other ways in which individuals within aggregated groups can interact with one another. In 
insects such as termites, pheromone mediated social interaction is thought to be important (Watanabe et al. 2014), but this does not appear to be the case in higher animals such as humans. The emerging field of "social signal processing" (a new cross-disciplinary research domain that aims at understanding and modelling social interactions) has begun to identify the many subtle ways in which human beings communicate non-verbally such as with gesture, interpersonal distance, posture and mutual gaze (Vinciarelli et al. 2012), but there are still many unanswered questions about how group propagative phenomena operate. A recent review suggests that a common, but poorly understood, principle of "density-dependent movement" (where clumping of individuals depends on the density of the group) might be involved in group aggregation across species (Liu et al. 2016). We suggest that, in higher animals, the role of the highly folded brain surface in facilitating such processes is worthy of further investigation.

\section{The brain, a delicate and easily-damaged organ, is in an exposed anatomical position}

The location of the brain is curious from the perspective of its extreme vulnerability. It is the control centre for human consciousness, cognition, peripheral organ function and homeostasis and has a delicate gelatinous consistency. It is therefore strange that evolution has resulted in it being one of the extremities of the body rather than being safely housed centrally (e.g. within the bony rib cage), as is the heart. Its vulnerability is underlined by the fact that, in bicycle accidents and other falls, the most common cause of death is brain injury (Thompson and Rivara 2001, Kim et al. 2000, Sterling et al. 2001). The brain is particularly exposed in bipedal humans, but head trauma is also a common cause of injury and death in other animals (Schwenk et al. 2016, Kuo et al. 2018). A need for proximity to highly prioritised sensory organs such as the eyes (Hofman 2014) seems an insufficient explanation: within the head, the visual cortex is as far away from the eyes as possible (Song et al. 2015) and long nerves, travelling some distance between the brain and other organs yet allowing rapid communication, are common in the body. The vagus nerve, that provides a highly responsive connection between the brain and the heart, is a good example (Idiaquez et al. 2018). Surely evolutionary pressures would have selected individuals with brains more centrally tucked away, unless there were other advantages to the brain's exposed location. One function for which this top-of-the-body location would be ideal is communication. The evolutionary pressures resulting in the transition from quadrupedal to bipedal locomotion in humans are not fully understood (Niemitz 2010), but have resulted in a body that looks rather like an antenna. Were the brain surface to have a communicative function in herd activity, this upright posture might be beneficial. Although many wave-forms can penetrate solid matter (e.g. the bony skull or the solid bodies of other members of the herd), the shape and location of wave emitters and receivers is important in improving performance: e.g. microphone shape and location is important in directionality which, in turn, is important in allowing localization of sensory input (Chung et al. 2008). 


\section{What happens if the brain's fractal geometry is impaired?}

Recent studies of the fractal geometry of the brain have shown that reduced surface area and folding are associated with severe neurodevelopmental problems, such as Schizophrenia and William's Syndrome (a chromosomal disorder with unique behavioural features) (Fahim et al. 2012). These disorders interfere with social communication, e.g. with the "serve and return" interaction that is characteristic of human verbal and non-verbal communication (Fahim et al. 2012, University 2015). At a microscopic level, problems in cerebellar Purkinje cell development are also related to disorders affecting social communication (Bauman and Kemper 2005). In Autism, a disorder characterised by problems with language and social communication, there is reduced brain surface area in the orbitofrontal cortex and reduced function/surface area in the left prefrontal cortex - two of the areas of the brain known to be most important in the more sophisticated aspects of social interaction (Happe et al. 1996) - and the development of brain surface area differs from typically developing children across the lifespan (Mensen et al. 2017). In addition to experiencing obvious social challenges, individuals with Autism can be more subtly challenged as regards group behaviour - for example being relatively immune to socially propagated yawning (Helt et al. 2010). A lack of response to such subtle group propagative phenomena might be evidence of impaired "herd" activity: these responses might not be social in the traditional sense (using the definition of social: "needing companionship") but could be social in terms of "relating to...social organisation" (Oxford 2016) i.e. predominantly functioning at an emotional or an automatic level.

\section{Might consciousness be shared across brains to enhance social organisation?}

Cacioppo has suggested that consciousness (defined as "the state of being aware of and responsive to one's surroundings") (Oxford 2016) is only useful, i.e. becomes more than simply an epiphenomenon of cognitive processes, if more than one brain is involved in social interaction. He uses the example of a person walking along a smooth street and suddenly encountering a hole in the road that causes him to exclaim. That exclamation serves no purpose. However should another person come around the corner at that moment, the exclamation might have various effects on the other person who may themselves exclaim, may apologise or may behave aggressively. This second person's reaction will, in turn, have an effect on the first person's actions and train of thought. Cacioppo's hypothesis is that consciousness needs to be shared with others in order to be useful. Cacioppo goes on to suggest that language mediates between these individual consciousnesses (Cacioppo and Decety 2011), but the sharing of consciousness goes beyond language and can be shared in pre-linguistic species that also use social interaction and attachment behaviours to increase community effectiveness and safety. We suggest that one location for sharing of consciousness might be the surface of the brain.We do not claim to understand how consciousness might be shared across brains. It is particularly challenging in this area that "there is, as yet, no universally agreed definition of consciousness" (Owen 2013) and neither is there certainty about the location of consciousness within the brain" (Owen 2013). Preliminary evidence from a small study of patients in vegetative/minimally conscious states has suggested that selective injuries to 
the neo-cortex, at the surface of the cerebral hemispheres - such as might result from hypoxic-ischemic encephalopathy - can result in greater loss of conscious awareness than more diffuse brain injuries in which the neo-cortex is relatively preserved (Cruse et al. 2012). In severe hypoxic-ischemic injuries damage to the neo-cortex and/or the thalamus is almost universal, probably due to greater oxygen demands in these brain regions: where there is thalamic damage, this renders the cortex effectively non-functional due to the lack of thalamic connections between different cortical areas (Adams et al. 2000). Experiments involving sleep and anaesthesia have also suggested that consciousness is related to this "cortical effective connectivity" (Ferrarelli et al. 2010).

\section{Biologically plausible processes are now recognised that could explain brain- brain synchrony}

Most traditional theories of consciousness within the brain could not be extrapolated to synchronous consciousness across more than one brain (e.g. Lutkenhoff et al. 2015). An exception - as plausible across two brains as within one - is that proposed by Penrose and Hameroff (Hameroff 2012). Their thinking is based on Quantum Field Theory (QFT), in which the fundamental building blocks of the universe are fields rather than particles (Hobson 2013). According to QFT, a quantum is a unit of energy that is discrete (i.e. "countable") yet connected to the entire field. Adding a quantum of energy to a field adds energy to the entire field yet that energy will be "superposed" upon quanta of the same frequency so that they can become "spatially bunched and in this sense more localized, but they are always of infinite extent" (Hobson 2013, p. 214). Quanta that are at a distance from one another can be "entangled", i.e. "they cannot be considered as separate independent objects - until they are finally disentangled by measurement" (Penrose 1995, p. 294). This has been demonstrated by "two slit experiments" in which the act of measurement appears to determine where a quantum of energy ends up in space. Considered in terms of fields rather than particles, this is easier to understand: a field of energy interacts with both slits simultaneously but, because particles are all-ornothing phenomena, the interaction with the detector will occur in a single, local area of the detection screen. This interaction is called a "collapse" - and because it is impossible to predict at which part of the detecting screen the interaction will occur, it is called "non-local collapse". If quanta were solely considered as particles, this would appear to be beyond reason - the single particle would appear to localise itself according to where the detector was placed (Hobson 2013). Einstein termed this "spooky interactions at a distance" (Hobson 1998). If, however, quanta are considered to be packets of energy which interact with a detector in an all-or-nothing way yet are connected infinitely to a field, then these interactions appear less spooky. There is now much interest in, and rapidly accruing evidence for, quantum phenomena in biology. Quantum phenomena have been demonstrated in photosynthesis, avian navigation systems and intracellular biological reactions (Lambert et al. 2013). In each of these systems, quantum processes seem to increase efficiency: harnessing the sun's energy for biological use with minimal energy loss; allowing birds to detect the earth's weak magnetic field and facilitating ultra-fast processes within cell enzymes and other proteins. Long-range electron transfer within intracellular proteins strongly suggests that quantum processes might be involved - but 
whether or not these proteins are developed to facilitate these quantum processes is unknown (Lambert et al. 2013). Hameroff and Penrose suggest that such protein specialisation, facilitating quantum processes, might be true for Tubulin, the protein that forms the basis of microtubules - a fundamental structural component of neurons (Hameroff and Penrose 1996). Their theory postulates that, across potentially wide brain regions, there is entanglement across the microtubular network and that this allows the ultra high-speed processes characteristic of consciousness. Specifically, the core protein component of the microtubule, tubulin, can exist in multiple superpositions of quantum states that interact by entanglement and then collapse to a definite state. Hameroff and Penrose termed this "objective reduction" (Hameroff and Penrose 1996) and go on to suggest that these episodes of collapse are not random, but are orchestrated by synaptic inputs and other factors. There is now evidence that networks of microtubules, examined in-vitro, can interact as a "colony" by self-organizing to produce higher-level phenomena (Hameroff 2012). Recently, it has been demonstrated that bundles of brain MTs spontaneously generate bursts of electrical activity and oscillations (del Rocio Cantero et al. 1980). It is a well established phenomenon of quantum physics that electrons do not respect physical barriers. In 1980, Don Devault stated that:

- 'Tunnelling' is the metaphorical name given to the process, possible in quantum mechanics, but not in classical mechanics, whereby a particle can disappear from one side of a potential-energy barrier and appear on the other side without having enough kinetic energy to mount the barrier. One can think of this as a manifestation of the wave-nature of particles (Devault 1980, p. 564)." Don Devault

If the quantum processes described by Penrose and Hameroff can account for entanglement across wide areas of a single brain, there is no reason why they cannot account for entanglement across more than one brain. Although the Penrose-Hameroff theory of consciousness has not yet been proven, the rapidly advancing fields of inter-brain synchrony (Dumas et al. 2010) and quantum biology (Lambert et al. 2013) and might suggest avenues for doing so.

\section{Impact}

Investigation of brain-brain surface interaction is likely to help us better understand important human phenomena such as parent-child interaction and social communication, ushering in the era of two-person neuroscience. When more sophisticated technologies are available to examine the way many brains might interact in networks, we may begin to understand group phenomena as disparate as sense of belonging, radicalisation and zeitgeist formation.

\section{Conclusion}

The brain is highly folded at its surface and, like other highly folded fractal structures in nature, it might have an important exchange function in communication at its surface. It is 
positioned in a vulnerable location in the body, especially in humans, but a location that might be ideal for a role in group interaction. Group processes in all species, including humans, goes beyond verbal and visual interaction. We propose that the structure and location of the brain surface might support quantum biological processes that allow shared consciousness and facilitate group cohesion.

\section{Competing Interests}

None of the authors have competing interests.

\section{Acknowledgements}

We are grateful to Justin Schniederman and Anthea McKinley for comments on earlier drafts and to Irene O'Neill for help with referencing.

\section{References}

- $\quad$ Adams JH, Graham D, Jennett B (2000) The neuropathology of the vegetative state after an acute brain insult. Brain 123 (7): 1327-1328. https://doi.org/10.1093/brain/ 123.7.1327

- $\quad$ Andrieu J, Hendry S, Hausberger M, Thierry B (2016) Informed horses are influential in group movements, but they may avoid leading. Animal Cognition 19 (3): 451-458. https://doi.org/10.1007/s10071-015-0945-2

- $\quad$ Batchelor G, Smith CA, Hill DL, Hawkes DJ, Cox TC, Dean AF (2002) Measures of folding applied to the development of the human fetal brain. IEEE Transactions on medical imaging. IEEE Transactions on Medical Imagining 21 (8): 953-965. https:// doi.org/10.1109/TMI.2002.803108

- Bauman ML, Kemper TL (2005) Neuroanatomic observations of the brain in autism: a review and future directions. International Journal of Developmental Neuroscience 23: 183-187. https://doi.org/10.1016/j.ijdevneu.2004.09.006

- Bevilacqua D, Davidesco I, Wan L, Chaloner K, Rowland J, Ding M, Rowland J, Michalareas G, Van Bravel J, Ding M (2019) Brain-to-brain synchrony and learning outcomes vary by student-teacher dynamics: Evidence from a real-world classroom electroencephalography study. Journal of Cognitive Neuroscience 31 (3): 401-411. https://doi.org/10.1162/jocn_a 01274

- Bowlby J (1982) Attachment and Loss. 2nd Edition, Vol. 1. Hogarth Press, London.

- Cacioppo JT, Decety J (2011) Social neuroscience: challenges and opportunities in the study of complex behavior. Annals of the New York Academy of Sciences 1224:

162-173. https://doi.org/10.1111/j.1749-6632.2010.05858.x

- Chaumont J, Guyon N, Valera AM, Dugue GP, Popa D, Marcaggi P, Gautheron V, Reibel-Foisset S, Dieudonne S, Stephan A (2013) Clusters of cerebellar Purkinje cells control their afferent climbing fiber discharge. Proceedings of the National Academy of Sciences 110 (40): 16223-16228. https://doi.org/10.1073/pnas.1302310110 
- $\quad$ Chung K, Neuman AC, Higgins M (2008) Effects of in-the-ear microphone directionality on sound direction identification. The Journal of the Acoustical Society of America 123 (4): 2264-2275. https://doi.org/10.1121/1.2883744

- $\quad$ Cruse D, Chennu S, Chatelle C, Fernandez-Espejo D, Bekinschtein TA, Pickard JD, Laureys S, Owen A (2012) Relationship between etiology and covert cognition in the minimally conscious state. Neurology 78 (11): 816-822. https://doi.org/10.1212/WNL. 0b013e318249f764

- del Rocio Cantero M, Etchegoyen CV, Perez PL, Scarinci N, Cantiello HF (1980) Bundles of brain microtubules generate electrical oscillations. Scientific Reports 8 (1): 11899.

- Devault D (1980) Quantum mechanical tunnelling in biological systems. Quartely Reviews of Biophysics 13 (4): 387-564. https://doi.org/10.1017/S003358350000175X

- Dikker S, Wan L, Davidesco I, Kaggen L, Oostrik M, McClintock J, Rowland J, Michalareas G, van Bravel J, Ding M, Poeppel D (2017) Brain-to-brain synchrony tracks real-world dynamic group interactions in the classroom. Current Biology 27 (9): 1375-1380. https://doi.org/10.1016/j.cub.2017.04.002

- Dumas G, Nadel J, Soussignan R, Martinerie J, Garnero L (2010) Inter-brain synchronization during social interaction. PLOS One 5 (8): e12166. https://doi.org/ 10.1371/journal.pone.0012166

- $\quad$ Fachner JC, Maidhof C, Grockie D, Nygaard Pedersen I, Trondalen G, Tucek G, Bonde LO (2019) '....telling me not to worry...' Hyperscanning and neural dynamics of emotion processing during Guided Imagery in Music. Frontiers in Psychology 10: 1561. https:// doi.org/10.3389/fpsyg.2019.01561

- Fahim C, Yoon U, Nashaat NH, Khalil AK, El-Belbesy M, Mancini-Marie A, Evans AC, Meguid N (2012) Williams syndrome: a relationship between genetics, brain morphology and behaviour. Journal of Intellectual Disability Research 56 (9): 879-894. https://doi.org/10.1111/j.1365-2788.2011.01490.x

- Ferrarelli F, Massimini M, Sarasso S, Casali A, Riedner BA, Angelini G, Tononi G, Pearce R (2010) Breakdown in cortical effective connectivity during midazolam-induced loss of consciousness. Proceedings of the National Academy of Sciences 107 (6): 2681-2686. https://doi.org/10.1073/pnas.0913008107

- Giroldini W, Pederzoli L, Bilucaglia M, Caini P, Ferrini A, Melloni S, Prati E, Tessoldi P (2015) EEG correlates of social interaction at distance. F1000Research 4.

- $\quad$ Giroldini W, Pederzoli L (2018) Brain-to-Brain Interaction at a distance based on EEG Analysis. Journal of Consciousness Exploration \& Research 9 (6): 501-513. URL: https://jcer.com/index.php/jcj/article/viewFile/757/762

- Hameroff S, Penrose R (1996) Orchestrated reduction of quantum coherence in brain microtubules: A model for consciousness. Mathematics and Computers in Simulation. 40: 453-480. https://doi.org/10.1016/0378-4754(96)80476-9

- Hameroff S (2012) How quantum brain biology can rescue conscious free will Frontiers in Integrative. Neuroscience Article 93: 1-17.

- Happe F, Ehlers S, Fletcher P, Frith U, Johansson M, Gillberg C, Dolan R, Frackowiak $R$, Frith C (1996) 'Theory of mind' in the brain. Evidence from a PET scan study of Asperger syndrome. Neuroreport: An International Journal for the Rapid Communication of Research in Neuroscience. 8 (1). 
- Hari R, Kujala MV (2009) Brain basis of human social interaction: from concepts to brain imaging. Physiological Reviews 89 (2): 453-479. https://doi.org/10.1152/physrev. $\underline{00041.2007}$

- Helt MS, Eigsti M, Snyder PJ, Fein DA (2010) Contagious yawning in autistic and typical development. Child Development 81 (5): 1620-1631. https://doi.org/10.1111/j. 1467-8624.2010.01495.x

- Hobson A (2013) There are no particles, there are only fields. American Journal of Physics 81: 211-223. https://doi.org/10.1119/1.4789885

- $\quad$ Hobson MP (1998) Letter from Einstein to Max Born, 3 March 1947; The Born-Einstein Letters; Correspondence between Albert Einstein and Max and Hedwig Born from 1916 to 1955. Quantum Entanglement and Communication Complexity. Walker, New York, CiteSeerX: 0.1.1.20.8324. pp.

- Hofman MA (2014) Evolution of the human brain: when bigger is better. Front Neuroanat 8 (15): 3389.

- Idiaquez J, Benarroch E, Nogues M (2018) Anatomy, Physiology, and Pharmacology of the Autonomic Nervous System (ANS). In: Idiaquez J, Benarroch E (Eds) Evaluation and Management of Autonomc Disorders: A Case Base Guide. Springer, Switzerland, 3-18 pp. https://doi.org/10.1007/978-3-319-7225-1

- Jiang X, Shen S, Cadwell CR, Berens P, Sinz F, Ecker AS, Patel S, Tolias AS (2015) Principles of connectivity among morphologically defined cell types in adult neocortex. Science 350 (6264): aac9462. https://doi.org/10.1126/science.aac9462

- Kimbell HS, Morrell LJ (2015) 'Selfish herds' of guppies follow complex movement rules, but not when information is limited. . Proceedings of the Royal Society, B 282 (1816): 2015558. https://doi.org/10.1098/rspb.2015.1558

- $\quad$ Kim KA, Wang MY, Griffith PM, Summers S, Levy ML (2000) Analyss of pediatric head injury from falls. Neurosurgical Focus 8 (1): 1-5. https://doi.org/10.3171/foc. 2000.8.1.154

- Krueger K, Flauger B, Farmer K, Hemelrijk C (2014) Movement initiation in groups of feral horses. Behavioural Processes 103: 91-101. https://doi.org/10.1016/j.beproc. 2013.10.007

- Kuo KW, Bacek LM, Taylor AR (2018) Head trauma. The Veterinary Clinics of North America Small Animal Practice 48 (1): 111-128. https://doi.org/10.1016/j.cvsm. 2017.08.005

- Lambert N, Chen Y-N, Cheng Y-C, Li C-M, Chen G-Y, Nori F (2013) Quantum Biology. Nature Physics 9 (1): 10. https://doi.org/10.1038/nphys2474

- Liu Q, Rietkerk M, Herman PM, Piersma T, Fryxell JM, van de Koppel J (2016) Phase separation driven by density-dependent movement: a novel mechanism for ecological patterns. Physics of Life Reviews 19: 107-121. https://doi.org/10.1016/j.plrev. 2016.07.009

- Lutkenhoff ES, Chiang J, Tshibanda L, Kamau M, Kirsch M, Pickard JD, Laureys S, Owen A, Monti M (2015) Thalamic and extrathalamic mechanisms of consciousness after severe brain injury. Annals of Neurology 78 (1): 68-76. https://doi.org/10.1002/ana. $\underline{24423}$

- Mensen VT, Wierenga LM, van Dijk S, Rijks Y, Oranje B, Mandl RC, Mandl R, Durston S (2017) Development of cortical thickness and surface area in autism spectrum disorder. Neurolmage: Clinical 13: 215-222. https://doi.org/10.1016/j.nicl.2016.12.003 
- Mota B, Herculano-Houzel S (2015) Cortical folding scales universally with surface area and thickness, not number of neurons. Science 349 (6243): 74-77. https://doi.org/ $\underline{10.1126 / \text { science.aaa9101 }}$

- $\quad$ Muller V, Sanger J, Lindenberger U (2013) Intra-and inter-brain synchronization during musical improvisation on the guitar. PLOS One 8 (9): e73852. https://doi.org/10.1371/ journal.pone.0073852

- Niemitz C (2010) The evolution of the upright posture and gait-a review and a new synthesis. Naturwissenschaften 97 (3): 241-263. https://doi.org/10.1007/ s00114-009-0637-3

- Okie JG (2013) General models for the spectra of surface area scaling strategies of cells and organisms: fractality, geometric dissimilitude, and internalization. The American Naturalist 181 (3): 421-439. https://doi.org/10.1086/669150

- Owen AM (2013) Detecting consciousness: a unique role for neuroimaging. Annual Review of Psychology 64: 109-133. https://doi.org/10.1146/annurevpsych-113011-143729

- Oxford (2016) Oxford Dictionaires. Lexico Powered by Oxford - Oxford University Press. http://www.oxforddictionaries.com/definition/english/consciousness. Accessed on: 2019-8-29.

- $\quad$ Penrose R (1995) Shadows of the Mind. A search for the missing science of consciousness. Vintage Science, London, 447 pp. [ISBN 9780099582113]

- Roth G, Dicke U (2005) Evolution of the brain and intelligence. Trends in Cognitive Sciences 9 (5): 250-257. https://doi.org/10.1016/j.tics.2005.03.005

- $\quad$ Schwenk BK, Furst AE, Bischofberger AS (2016) Traffic accident-related injuries in horses. Pferdeheilkunde 32: 192-199. https://doi.org/10.21836/PEM20160301

- Song C, Schwarzkopf DS, Kanai R, Rees G (2015) Neural population tuning links visual cortical anatomy to human visual perception. Neuron 85 (3): 641-656. https://doi.org/ 10.1016/j.neuron.2014.12.041

- Sterling DA, O'Connor JA, Bonadies J (2001) Geriatric falls: injury severity is high and disproportionate to mechanism. Journal of Trauma and Acute Care Surgery 50 (1): 116-119. https://doi.org/10.1097/00005373-200101000-00021

- Thompson MJ, Rivara FP (2001) Bicycle-related injuries. American Family Physician 63 (10): 2007-2014.

- $\quad$ Tsubota T, Ohashi Y, Tamura K (2013) Optogenetics in the cerebellum: Purkinje cellspecific approaches for understanding local cerebellar functions. Behavioural Brain Research 255: 26-34. https://doi.org/10.1016/j.bbr.2013.04.019

- University H (2015) Serve and Return - Centre on the Developing Child, Harvard University. http://developingchild.harvard.edu/science/key-concepts/serve-and-return/

- Vinciarelli A, Pantic M, Heylen D, Pelachaud C, Poggi I, D'Errico F, Schroeder M (2012) Bridging the gap between social animal and unsocial machine: A survey of social signal processing. IEEE Transactions on Affective Computing 3 (1): 69-87. https://doi.org/ 10.1109/T-AFFC.2011.27

- Watanabe D, Gotoh H, Miura T, Maekawa K (2014) Social interactions affecting caste development through physiological actions in termites. Frontiers in Physiology 5 https:// doi.org/10.3389/fphys.2014.00127

- West GB, Brown JH, Enquist BJ (1999) The fourth dimension of life: Fractual geometry and allometric scaling of organisms. Science 284 (5420): 1677-1679. https://doi.org/ $\underline{10.1126 / \text { science. } 284.5420 .1677}$ 